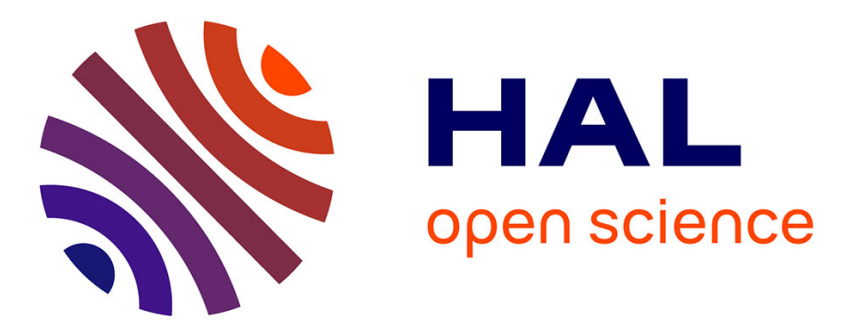

\title{
Quantitative insights into the growth mechanisms of nanopores in hexagonal boron nitride
}

Ki Kang Kim, Young Hee Lee, Ouafi Mouhoub, Rafael Martinez-Gordillo, Jaysen Nelayah, Guillaume Wang, Ji-Hoon Park, Young Hee Lee, Christophe Bichara, Annick Loiseau, et al.

\section{To cite this version:}

Ki Kang Kim, Young Hee Lee, Ouafi Mouhoub, Rafael Martinez-Gordillo, Jaysen Nelayah, et al.. Quantitative insights into the growth mechanisms of nanopores in hexagonal boron nitride. Physical Review Materials, 2020, 4 (1), 10.1103/PhysRevMaterials.4.014005 . hal-03034741v2

\section{HAL Id: hal-03034741 \\ https://hal.science/hal-03034741v2}

Submitted on 14 Dec 2020

HAL is a multi-disciplinary open access archive for the deposit and dissemination of scientific research documents, whether they are published or not. The documents may come from teaching and research institutions in France or abroad, or from public or private research centers.
L'archive ouverte pluridisciplinaire HAL, est destinée au dépôt et à la diffusion de documents scientifiques de niveau recherche, publiés ou non, émanant des établissements d'enseignement et de recherche français ou étrangers, des laboratoires publics ou privés. 


\title{
Quantitative insights into the growth mechanisms of nanopores in hexagonal boron nitride
}

\author{
Ouafi Mouhoub, ${ }^{1,2}$ Rafael Martinez-Gordillo, ${ }^{3}$ Jaysen Nelayah, ${ }^{1}$ Guillaume Wang, ${ }^{1}$ Ji-Hoon Park, ${ }^{4,5}$ Ki Kang Kim, ${ }^{6}$ \\ Young Hee Lee, ${ }^{4,5}$ Christophe Bichara ${ }^{3}$ Annick Loiseau, ${ }^{2}$ Christian Ricolleau, ${ }^{1}$ Hakim Amara $\odot,{ }^{1,2, *}$ \\ and Damien Alloyeau ${ }^{1, \dagger}$ \\ ${ }^{1}$ Laboratoire Matériaux et Phénomènes Quantiques, Université de Paris-CNRS, F-75013 Paris, France \\ ${ }^{2}$ Laboratoire d'Etude des Microstructures, ONERA-CNRS, UMR 104, \\ Université Paris Saclay, BP 72, 92322 Chatillon Cedex, France \\ ${ }^{3}$ Aix Marseille Université, CNRS, CINAM, 13288 Marseille, France \\ ${ }^{4}$ Center for Integrated Nanostructure Physics, Institute for Basic Science, Suwon 16419, Republic of Korea \\ ${ }^{5}$ Department of Energy Science, Sungkyunkwan University, Suwon 16419, Republic of Korea \\ ${ }^{6}$ Department of Energy and Materials Engineering, Dongguk University Seoul, Seoul 04620, Republic of Korea
}

(Received 30 July 2019; published 21 January 2020)

\begin{abstract}
The formation of nanopores under electron irradiation is an ideal process to quantify chemical bonds in two-dimensional materials. Nowadays, high-resolution transmission electron microscopy (HRTEM) allows investigating such nucleation and growth phenomena with incomparable spatial and temporal resolution. Moreover, theoretical calculations are usually exploited to confirm characteristic features of these atomic-scale observations. Nevertheless, the full understanding of the ejection mechanisms of atoms requires a detailed investigation of the interplay between the very dynamic edge structure of expanding nanopores and the displacement energy of edge atoms $\left(E_{D}\right)$. Here, the dynamics of triangular nanopores in hexagonal boron nitride (h-BN) under various electron dose rates was followed by aberration-corrected HRTEM with high temporal resolution to provide new in situ insights into their growth processes. We reveal that the ejection of atomic pairs is an elemental mechanism that considerably speeds up the expansion of nanopores. Atomic-scale calculations were exploited to quantify the structure-dependent $E_{D}$ of all the ejected edge atoms. They revealed strong variations of this threshold energy during the growth processes. This quantitative study reconciles theoretical and experimental measurements of the ejection rate of atoms in h-BN under electron irradiation, which is essential for nanopore engineering in this atomically thin membrane.
\end{abstract}

DOI: 10.1103/PhysRevMaterials.4.014005

\section{INTRODUCTION}

Given its resolution and single-atom sensitivity, aberrationcorrected high-resolution transmission electron microscopy (HRTEM) has been extensively exploited to reveal the atomic structure of two-dimensional (2D) materials [1-3]. Although radiation damage is an obvious limitation of HRTEM investigations [4], studying the dynamics of defects in $2 \mathrm{D}$ materials under beam irradiation gives access to quantitative information on their structural properties. In this respect, the beam-induced nucleation and growth of nanopores in graphene and its derivatives have become a case study to better understand the fragility of these beam-sensitive materials and the fabrication processes of nanosized holes in atomically thin membranes that are promising for gas sensing, DNA sequencing, and water desalinization technologies [5-13]. Furthermore, the spatial and temporal resolutions of HRTEM allow precise measurement of the expulsion rate of atoms as a function of the electron dose from which an average value of the displacement energy of atoms $\left(\overline{E_{D}}\right)$ can be calculated [14-16]. This threshold energy required to displace

\footnotetext{
*Corresponding author: hakim.amara@onera.fr

†damien.alloyeau@univ-paris-diderot.fr
}

an atom from its lattice can also be calculated with theoretical approaches using density functional theory (DFT) or coupling it with molecular dynamics [17-19]. In the case of hexagonal boron nitride (h-BN), ab initio calculations have shown that the displacement energy of an atom $\left(E_{D}\right)$ is element specific and very sensitive to its local atomic environment. They allowed explaining some features of the growth mechanisms of nanopores, such as the formation of boron monovacancies in a pristine area, the growth of nanopores via the preferential ejection of edge atoms, and the continuous reconstruction of triangular holes observed at room temperature $[6,14,18-21]$. Nevertheless, the structure dependency of $E_{D}$ in h-BN makes the interpretation of HRTEM measurements complicated because the strong variations of $E_{D}$ that occur during the growth processes are all integrated in these global measurements. Therefore investigating this dynamic interplay between the atomic structure and the energy profile of edge atoms in 2D materials is necessary to match experimental and theoretical measurements of $E_{D}$ and to provide a deeper understanding of nanopore growth mechanisms.

In the present paper, the dynamics of triangular nanopores in h-BN under various electron dose rates was followed with unprecedented time resolution. Besides confirming previously reported nucleation and growth mechanisms, these atomicscale observations provide new in situ insights into the ejec- 
tion mechanisms of atoms. DFT calculations were exploited to understand and support HRTEM results and to quantify the variations of $E_{D}$ during the growth processes that must be unraveled to understand the low $\overline{E_{D}}$ extracted from our experimental data.

\section{METHODS}

The h-BN sample was prepared by chemical vapor deposition synthesis on a polycrystalline platinum film by using borazine as a liquid precursor [22]. Briefly, a Pt foil (thickness of $0.1 \mathrm{~mm}$, Goodfellow) was mounted at the center of a quartz tube in chemical vapor deposition, and then the temperature was elevated up to $1100{ }^{\circ} \mathrm{C}$ for $30 \mathrm{~min}$ at a flow rate of 10 sccm of $\mathrm{H}_{2}$ gas. Monolayer h-BN was grown for $30 \mathrm{~min}$ with $\mathrm{H}_{2}$ and borazine at flow rates of 10 and $0.6 \mathrm{sccm}$, respectively. After the growth of h-BN, the furnace was cooled naturally. The bubbling transfer was exploited to transfer h-BN film onto TEM grids [23].

We used a JEOL atomic resolution microscope (ARM) $200 \mathrm{~F}$ at $80 \mathrm{kV}$ which combines a cold field-emission gun, an aberration corrector (CEOS, Inc.), and a high frame rate camera (Oneview, Gatan, Inc.) [24,25]. The pressure in the microscope was $10^{-7}$ mbar and we used a "cold finger" to keep contaminants away from the specimens. The sample was imaged with a positive spherical-aberration coefficient of $5 \mu \mathrm{m}$ and we maintain the focus close to Scherzer conditions (the focus can vary during long-time acquisition due to h-BN film oscillation). In these conditions, the atoms of single layer h-BN have a dark contrast with respect to the background, but the contrast of all the images and video has been numerically inverted to make them more intuitive. After identifying monolayer areas, the nucleation and growth of nanopores were monitored with a frame rate varying from 3 to 12 frames/s. These conditions are a good compromise to optimize the temporal resolution and image the structure of the h-BN monolayer with a sufficient signal to noise ratio. Image summing or Wiener filters were applied when necessary to reveal the structure of nanopores. These two numerical processes were used to improve the signal to noise ratio of images showing edge configurations with long and short lifetime, respectively. Note that some bright spots in the images are due to dead pixels that were unfortunately present on the camera when the data were collected. However, these artifacts can easily be identified on the image series (or videos) because their position remains stable in spite of sample drift and they can also be seen over vacuum.

To precisely measure the dose rate $(\dot{d})$, the conversion efficiency (i.e., number of counts per electron) of the One view camera was calibrated prior to inserting the sample, by measuring the current on the small phosphorescent screen of the microscope and the number of counts detected by the camera. In accordance with the specifications given by Gatan, we measured a conversion efficiency of 53.3 counts per electron at $200 \mathrm{kV}$ (52.4 according to Gatan) and 103.1 counts per electron at $80 \mathrm{kV}$. Thus, it is possible to determine the dose rate (in electrons $/ \mathrm{s}^{-1} \mathrm{~nm}^{-2}$ ) and the cumulative dose ( $d_{\mathrm{tot}}$, in electrons $/ \mathrm{nm}^{-2}$ ), by measuring the number of counts per second and per square nanometer over the vacuum (e.g., in the nanopores). In order to extract quantitative information on the structural properties of h-BN, we studied the formation of nanopores as a function of $\dot{d}$ ranging from $3.1 \times 10^{5}$ to $2.0 \times 10^{6}$ electrons $/ \mathrm{s}^{-1} \mathrm{~nm}^{-2}$.

To investigate the different mechanisms leading to the growth of nanopores, we performed $a b$ initio calculations within the DFT using the SIESTA code [26] with generalized gradient approximation exchange-correlation functionals. We used norm-conserving Trouiller-Martin pseudopotentials and a numerical atomic orbital with double- $\zeta$ plus polarization basis set to represent the valence electron with a real-space integration grid of $300 \mathrm{Ry}$ as in [27,28]. The system consists of a central nanopore of different sizes within a supercell sufficiently large to minimize boundary effects on the energies of interest. For pristine BN, we adopted a $(10 \times 10)$ grid of 200 atoms and the size of the supercell in the $c$ direction was set to $25 \AA$ to avoid the interaction between neighboring defects. It is important to note that we chose this system size after carrying out some comparisons using different supercells (144, 200, and 720 atoms) in order to evaluate the impact of the interaction between the images of structural defects due to the periodic boundary conditions. In the present paper, the grid cutoff used for the k-point sampling was $16 \AA$. Calculations were performed at zero pressure, i.e., allowing the relaxation of the atoms and the shape of the simulation cell, using a conjugate gradient minimization scheme. The atomic positions were relaxed until the magnitudes of the forces on all the atoms were smaller than $0.01 \mathrm{eV} / \AA$.

To calculate $E_{D}$ of an atom (B or $\mathrm{N}$ ), we assumed that this quantity is related to the formation energy of an interstitialvacancy pair as in [19,29] and performed static calculations as

$$
E_{D}=E\left(N_{\text {tot }}+1\right)+E\left(N_{\text {tot }}-1\right)-2 E\left(N_{\text {tot }}\right),
$$

where $E\left(N_{\text {tot }}\right)$ is the total energy of an initial configuration containing $N_{\text {tot }}$ atoms, and $E\left(N_{\text {tot }}+1\right)$ and $E\left(N_{\text {tot }}-1\right)$ are the total energies of the initial system where an atom is added and removed, respectively. Thereby, $E_{D}$ is defined as the minimal kinetic energy that has to be transferred to a lattice atom in order to create a stable Frenkel pair (interstitial-vacancy pair) [30]. Such an approach has been successfully employed to characterize irradiation effects on carbon nanotubes [29], BN sheets [19], and also bulk materials like $\mathrm{UO}_{2}$ [31]. Note that this equation does not account for the barrier separating the ideal and defective configurations and gives access to the lowest energy for the system to switch from an initial state to a final state whatever the intermediate steps involved in the process. Meanwhile, it is well known that this static approach used in our calculations gives a lower bound estimate of the damage threshold energies while molecular dynamics simulations can be used but they may overestimate $E_{D}[19,29,32]$. This artifact has already been discussed in detail for the rather simple case of the displacement energy in graphene. [17,29]. A molecular dynamics calculation gives a value of $22 \mathrm{eV}$, while a static calculation tends to $15 \mathrm{eV}$ [29]. On one hand, the molecular dynamics result is in line but somewhat larger than the values of 15-20 eV reported in experimental studies for graphite [33]. On the other hand, the static calculations agree with the experimental data, being on the lower side of the data distribution. One can expect that the correct value of $E_{D}$ is between both approaches. However, the most important result is 
(a)

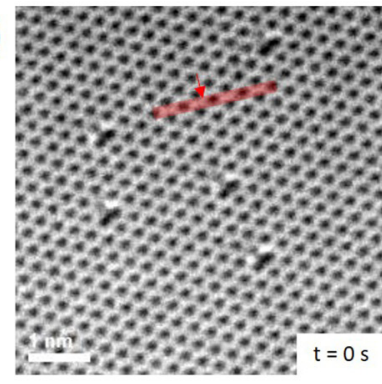

(c)

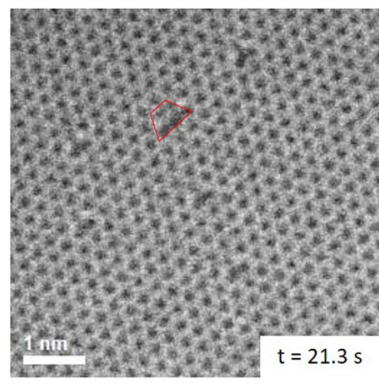

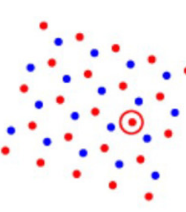
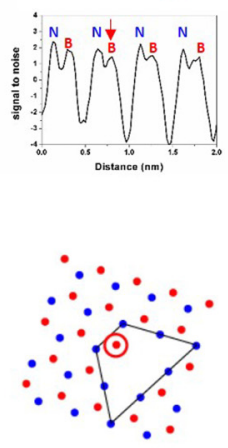

(b)

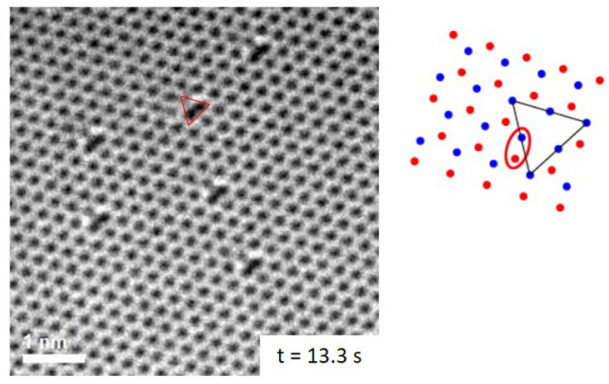

(d)

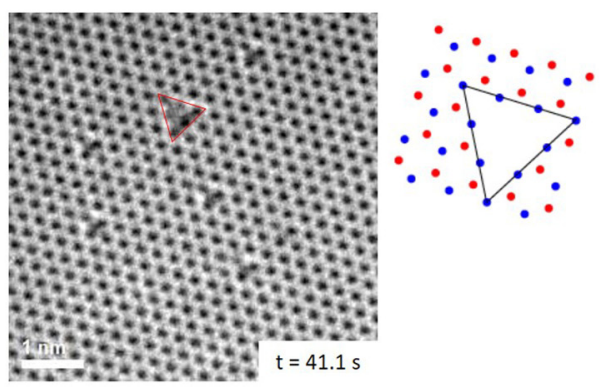

FIG. 1. Nucleation of nanopores. HRTEM images are extracted from a movie acquired with a frame rate of 3 frames/s that can be seen in Supplemental Material [34]. A model of the observed atomic structure is presented next to each image. Boron atoms are in red and nitrogen atoms are in blue. The atom(s) encircled are ejected in the next frame. The signal-to-noise-ratio profile next to the image (a) was measured along the red line seen in image (a). It shows that the missing atom in frame (b) was a B atom.

that a static approach yields meaningful physical information, keeping in mind that the calculated displacement energies might be slightly underestimated. Thus, the main contribution characterizing the displacement of atoms may come from the differences between their binding energies, making the use of static calculations relevant. In the case of hBN (pristine and nanopores), our results of displacement energies from static calculations are given in Table S1 in Supplemental Material [34]. They are in good agreement with values found in [19] and references therein using other approaches. Moreover, in the case of the ejection of a $\mathrm{B} / \mathrm{N}$ pair, the same equation can be used by considering a pair added or removed from the initial system composed of $N_{\text {tot }}$ atoms.

\section{RESULTS AND DISCUSSION}

We have exploited aberration-corrected HRTEM to study the nucleation and growth mechanisms of nanopores in single layer h-BN. The analysis of single-atom contrasts performed before the nucleation processes revealed that monovacancies are generated by the ejection of B atoms [Figs. 1(a) and 1(b)]. The common orientation of triangular patterns generated by these single point defects confirms that all monovacancies have the same structure because the ejection of single B and single $\mathrm{N}$ atoms would generate triangular patterns rotated by $180^{\circ}$ relative to each other. As observed in Figs. 1(b)-1(d), some monovacancies transform into triangular nanopores with four missing atoms ( $3 \mathrm{~B}$ and $1 \mathrm{~N}$ ). This transformation passes through an intermediate structure with a trapezoidal shape after the ejection of a B-N pair [Fig. 1(c) and Fig. S1(a) in Supplemental Material [34]]. These trapezoidal nanopores are intermittently observed for a few seconds because of an important edge mobility that highlights the poor stability of this intermediate step. Finally, the ejection of a last B atom generates a triangular hole with edges in zig-zag configuration and $\mathrm{N}$ atoms at the forefront of the edges. This characteristic structure of nanopores in h-BN undoubtedly presents a high energetic stability, because the atomic mechanisms that occur during the subsequent growth processes systematically tend towards this configuration. As shown in Fig. 2, the growth of nanopores is a two-step mechanism which starts with the ejection of edge atoms creating a notch on one side of the triangular hole. These notched edges, which expose boron atoms at the rim of the hole, are unstable intermediate structures with a reduced lifetime $(<1 \mathrm{~s})$ compared to zig-zag edges without defect that can resist the electron beam for several seconds. Indeed, just after this first step, we systematically observe a peeling of the notched edges via the gradual ejections of atoms on both sides of the notch. This second step leads to the formation of a larger triangular hole with a zig-zag edge that is shifted outward by $0.25 \mathrm{~nm}$ as compared to the initial triangular nanopores.

All these HRTEM observations are consistent with previous works, notably the extended study of Ryu et al. [6]. Nevertheless, the high-speed acquisition used in the present paper brings additional mechanistic information on the growth process. At first, the ejections of individual $\mathrm{B}$ or $\mathrm{N}$ atoms from the edges of nanopores were never observed even when the nanopores dynamics was followed with a frame rate of 12 images per second. Most of the time, the first image of a notch shows the ejection of a pair of B-N atoms from the edge [Fig. 2 and Fig. S1(b) in Supplemental Material [34]]. The analysis of the notch position performed over 75 events clearly demonstrates that these defects are mainly formed in the central region of triangle sides (Fig. S2 in Supplemental 

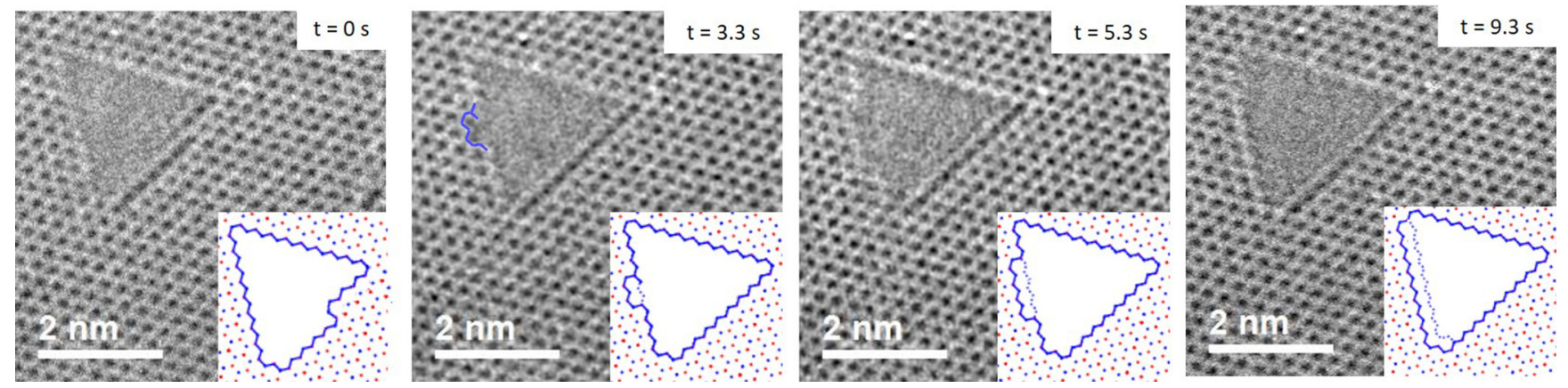

FIG. 2. Growth of nanopores. HRTEM images extracted from a movie acquired with a frame rate of 3 frames/s that can be seen in Supplemental Material [34]. A model of the observed atomic structure and the time frame are shown in the right bottom and top corner of each image, respectively. Boron atoms are in red and nitrogen atoms are in blue.

Material [34]) and the first atom pair ejected is never part of the nanopore corners. Interestingly, the peeling mechanism also implies the ejection of B-N pairs (Fig. 3). The lifetime of the B-N pairs adjacent to the growing notch varies from a few tenths of second to a few seconds. The previously reported formation and ejection of atom chains were never observed in our experimental conditions [6].

Obviously, increasing the electron dose rate speeds up the growth of nanopores, but it also modifies their growth mechanisms because it affects the competition between the formation of notches and the peeling process. Indeed, when higher dose rates are used, deeper notches with two or three steps can be observed, because B-N pairs are ejected from the lower edge before the peeling of the upper edge is completed (Fig. S3 in Supplemental Material [34]). These deeper notches drastically increase the growth kinetics because the subsequent peeling processes induce the ejection of several layers of atoms. The presence of these defects increases the edge roughness. Therefore, the decrease of the averaged convexity of nanopores when the dose rate increases (Fig. S4 in Supplemental Material [34]) demonstrates that high dose rate favors the formation of multistep notches. As the probability of notch formation and the duration of the peeling process increase with the size of nanopores, multistep notches are also more frequent in large nanopores. Interestingly, with a dose rate ranging from $3.1 \times 10^{5}$ to $2 \times 10^{6}$ electrons $/ \mathrm{s}^{-1} \mathrm{~nm}^{-2}$, multistep notches remain rare in small nanopores (perimeter $<9 \mathrm{~nm}$ ). It is worth noting that the growth of nanopores is also drastically impacted by coalescence phenomena that affect both the size and the density of nanopores (Fig. S5 in Supplemental Material [34]). In a general manner, the engineering of nanopores in $\mathrm{h}-\mathrm{BN}$ cannot disregard the crucial effects of coalescence and multistep notches on the density and final size of holes.
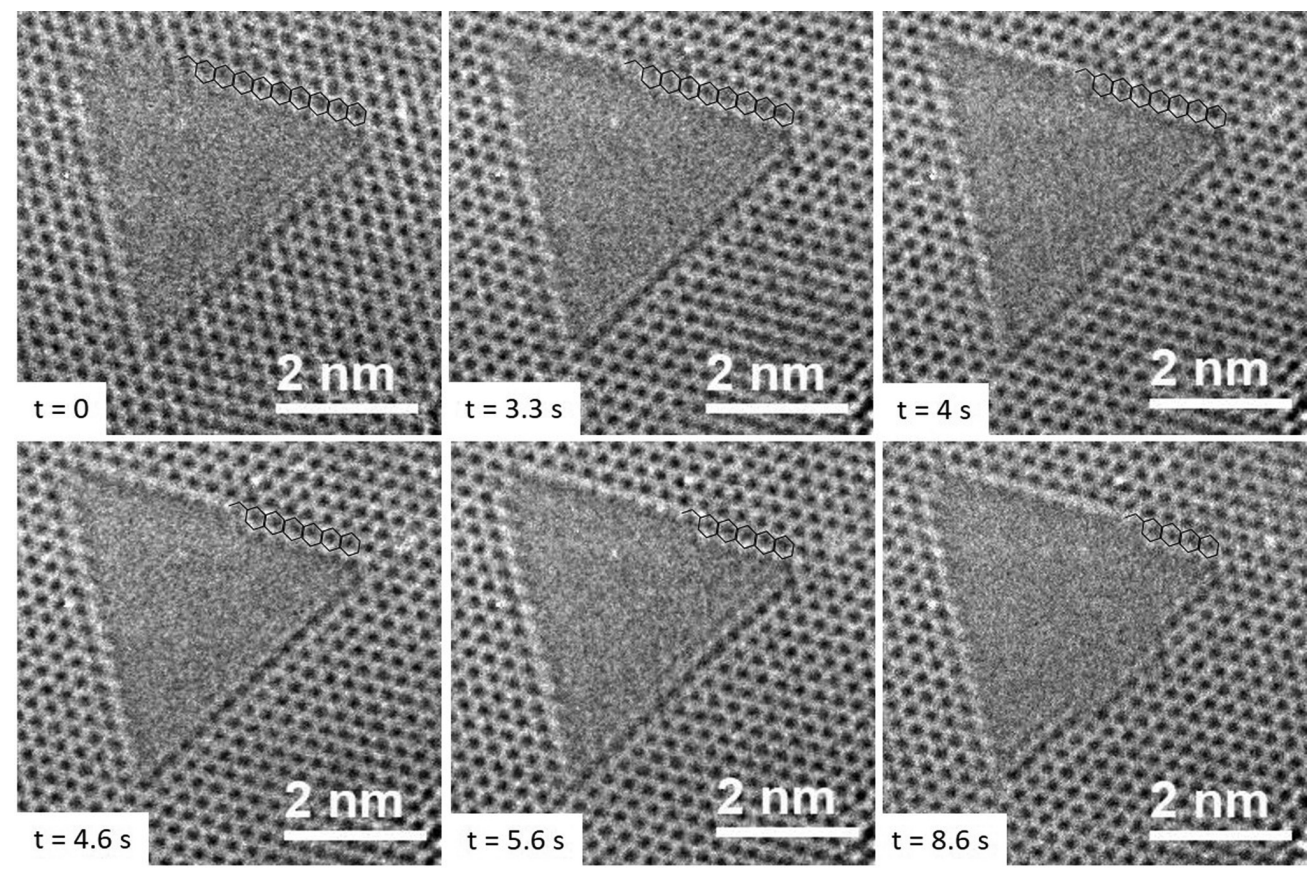

FIG. 3. Atom-pair ejection during the peeling process. HRTEM images extracted from a movie acquired with a frame rate of 3 frames/s that can be seen in Supplemental Material [34]. The time frame is shown in the right bottom corner of each image. The atomic lattice of the top edge is highlighted. No intermediate structure is observed between frames. 

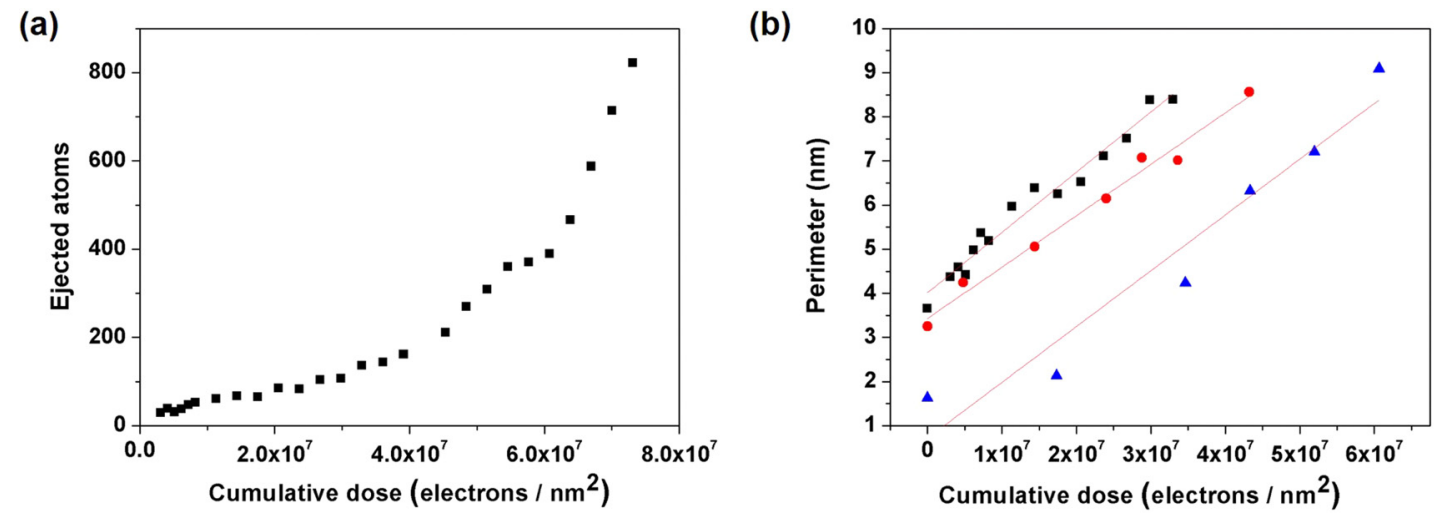

FIG. 4. Growth of individual nanopores without a coalescence event. (a) Number of ejected atoms as a function of the cumulative dose with a dose rate of $3.1 \times 10^{5}$ electrons $/ \mathrm{s}^{-1} \mathrm{~nm}^{-2}$. (b) Perimeter of nanopores as a function of the cumulative dose with a dose rate of $3.1 \times$ $10^{5}$ electrons $/ \mathrm{s}^{-1} \mathrm{~nm}^{-2}$ (black squares), $4.8 \times 10^{5}$ electrons $/ \mathrm{s}^{-1} \mathrm{~nm}^{-2}$ (red dots), and $8.5 \times 10^{5}$ electrons $/ \mathrm{s}^{-1} \mathrm{~nm}^{-2}$ (blue triangle).

In the following, we use our atomic scale observations to measure the average displacement energy of atoms at the edge of nanopores $\left(\overline{E_{D}^{\text {ed }}}\right)$ and compare it with ab initio calculations. This fundamental understanding of h-BN structural properties requires one to focus on small nanopores that grow without coalescence event and multistep notches. Determining $\overline{E_{D}^{\mathrm{ed}}}$ requires measuring the cross section for removing edge atoms $\left(\sigma^{\text {ed }}\right)$, which is given by

$$
\sigma^{\text {ed }}=\frac{N}{n \times d}
$$

with $N$ the number of ejected atoms, $n$ the number of irradiated edge atoms, and $d$ the cumulative dose in electrons per square nanometer. As $\sigma$ is a geometrical approximation of the ejection rate of atoms, it is obviously a constant. However, in our experiments, $N$ is not directly proportional to $d$ [Fig. 4(a)], because $n$ increases over time as the nanopores grow. By considering that nanopores have a triangular shape, we can obtain a more useful expression of $\sigma$ :

$$
\sigma^{\mathrm{ed}}=\frac{D}{12 \sqrt{3} D_{e}} \times \frac{P}{d}
$$

with $D$ the surface atom density of h-BN $\left(38\right.$ atom $\left./ \mathrm{nm}^{2}\right), D_{e}$ the atomic density along the edge of nanopores $(8$ atom $/ \mathrm{nm}$ for edges in zig-zag configuration), and $P$ the perimeter of nanopores (see Supplemental Material [34]). As observed in Fig. 4(b), for small nanopores $(P<9 \mathrm{~nm}), P$ varies linearly with $d$ and the $P / d$ ratio is dose-rate independent and similar for all the nanopores that grow without coalescence phenomena. This linear relationship arises from the fact that the growth of nanopores is driven by the ejection of edge atoms. The average $P / d$ ratio measured over eight nanopores obtained with five different dose rates is $3.55 \pm$ $0.8 \mathrm{~nm}^{3} /$ electron, leading to $\sigma^{\text {ed }}=241 \pm 54$ barns. By using the model of Seitz and Koehler (see $[15,16,35]$ ) we calculate that $\overline{E_{D}^{\text {ed }}}$ is between 3.9 and $5.3 \mathrm{eV}$ (see Supplemental Material [34] including Fig. S6).

DFT calculations were performed to determine the displacement energies of atoms at the edge of nanopores and to confirm the growth mechanisms observed by HRTEM. In the following, we consider a system consisting of a BN sheet containing 200 atoms with a nanopore of nine missing atoms [Fig. 5(a)], with periodic boundary conditions. Regarding the notch formation on zig-zag edges, our DFT calculations performed on a relaxed triangular nanopore confirm that the displacement of atoms by a B-N pair $(12.46 \mathrm{eV})$ is energetically more favorable than the sequential displacement of $\mathrm{N}$ first and then $\mathrm{B}$ atoms $(7.85+10.32 \mathrm{eV})$ and the opposite, $\mathrm{B}$ first and then $\mathrm{N}$ atoms $(13.03+4.35 \mathrm{eV})$ (Fig. 5 and Fig. S7 in Supplemental Material [34]). Moreover, our simulations show that the notch formation is mainly located at the central part of the triangle side. Indeed, a significant increase of $E_{D}^{\text {ed }}$ was calculated when the ejected B-N pair lies closer to the nanopore corners (Fig. S8 in Supplemental Material [34]). It is worth noting that calculations performed on larger nanopores (16 and 25 missing atoms) lead to similar conclusions and showed no significant effect of the pore size on the value of $E_{D}^{\text {ed }}$ (Figs. S9 and S10 in Supplemental Material [34]). Thus, the system considered subsequently is large enough to safely investigate the growth mechanisms of nanopores. After the notch formation, we now focus on the peeling process leading to the formation of a triangular nanopore with 16 missing atoms. Our DFT calculations also confirm the second step of the growth processes observed by HRTEM. Figure 5 shows the successive ejections of the least stable atom pairs. Several possibilities of B-N pair ejections were explored at each step (Fig. S11 in Supplemental Material [34]). Systematically, the atomic pairs adjacent to both sides of the notch are preferentially ejected with an $E_{D}^{\text {ed }}$ of $10.60 \mathrm{eV}$ on average [Figs. 5(c) and 5(d)]. The last step to form a regular triangle with corners in armchair configuration involves the ejection of a single boron atom which requires $10.19 \mathrm{eV}$ [Fig. 5(e)]. The ejection of B-N pairs at the edge of nanopores necessarily implies the breaking of a chemical bond perpendicular to the edge. In line with the study of Ryu et al. [6], DFT calculations of relaxed nanopores (Fig. S12 in Supplemental Material [34]) show that the chemical bonds perpendicular to the edges are the longest $(0.158 \mathrm{~nm})$, which makes them more fragile than the bonds along the edges (with a length below $0.152 \mathrm{~nm}$ ) or the bonds in the nanopore corners, which are the shortest (below $0.141 \mathrm{~nm}$ ). We assume that these weaker bonds play a key role in the atomistic processes leading to the ejection of paired atoms. 


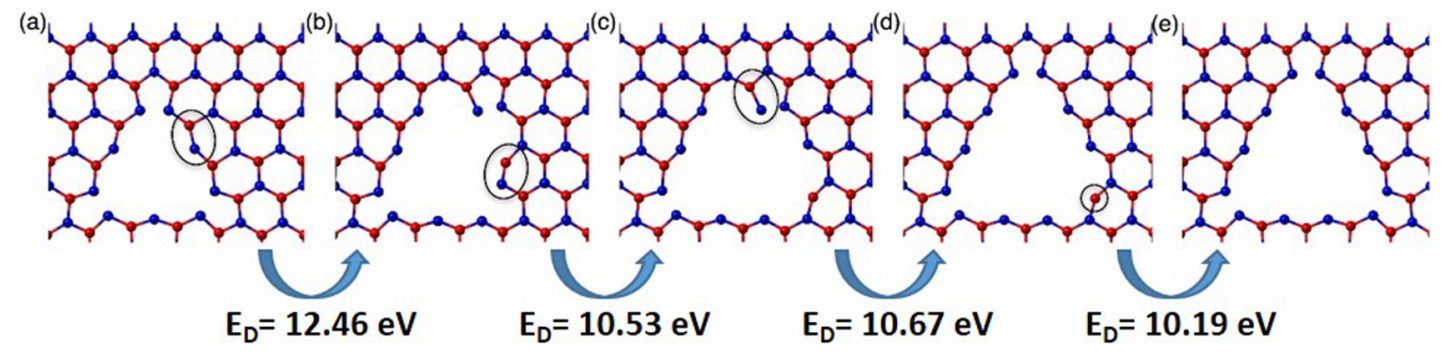

FIG. 5. DFT calculations. Growth mechanism of an individual nanopore with nine missing atoms. The displacement energy of the ejected atoms (encircled) is given at each step. (a)-(d) B-N pairs are ejected. (d), (e) A single B atom is ejected. (e) The final structure corresponds to a nanopore with 16 missing atoms. Boron atoms are in red and nitrogen atoms are in blue. All the calculations performed to obtain this energetically favorable growth mechanism are described in Fig. S11 in Supplemental Material [34].

All these conclusions are in agreement with the growth sequence observed experimentally involving the higher stability of zig-zag edges without notch and the fast ejection of pairs of atoms from the center of the triangle side to the apex once a notch is formed. Additionally, our calculations provide valuable information on the energies involved throughout the process. Indeed, it is then possible to extract from this analysis the average $\overline{E_{D}^{\text {ed }}}$ for nanopores growing from a perimeter of 2.25 to $9 \mathrm{~nm}$. By considering successive notch nucleation (two atoms with $E_{D}^{\text {ed } ~}=6.23 \mathrm{eV} /$ atom) and expansion $\left(5.3 \mathrm{eV} /\right.$ atom + one atom with $\left.E_{D}^{\mathrm{ed}}=10.19 \mathrm{eV}\right)$, we found that $\overline{E_{D}^{\text {ed }}}$ is equal to $5.8 \mathrm{eV}$, a slightly larger value than the experimental estimate. Therefore, the fair agreement between our experimental and theoretical investigations confirms that the ejection of atom pairs is the elementary mechanism of the nanopore growth process in h-BN. Moreover, we show that a correct determination of the displacement energy requires considering separately the formation of the notch and its expansion. It can be noted that our experimental and theoretical measurements of $\overline{E_{D}^{\mathrm{ed}}}$ are performed on small nanopores because the multistep notches observed in larger pores would induce further variations of the energy profile of edge atoms that would very likely reduce $\overline{E_{D}^{\mathrm{ed}}}$. A complete quantitative study of the growth processes of nanopores in h-BN should take into account the effect of these more complex structural configurations together with the drastic influence of coalescences processes. Other parameters such as the presence of adsorbates and impurities, the residual pressure and composition of the gaseous environment around the sample, or inelastic irradiation damages can also influence the formation kinetics of nanopores and could explain the discrepancies observed in the literature [14]. Notably, the presence of single metal or Si atoms on 2D materials, combined with residual oxygen in the TEM column, can speed up the formation of nanopores by catalytic etching [36,37]. Nevertheless, the sensitivity of HRTEM imaging provides the opportunity to unambiguously detect the presence of single heavy atoms on 2D materials [36,38]. In our experimental conditions (see method section), the absence of strong-atomic contrast on the $\mathrm{h}-\mathrm{BN}$ and the very good agreement between the experimental and theoretical growth rates of nanopores strongly indicate that the observed atomic processes are dominantly driven by knock-on damage.
To enhance the validity of this conclusion and to evaluate the nucleation rate of nanopores, we have also determined both experimentally and theoretically the displacement energy of single $\mathrm{B}$ atoms in pristine h-BN $\left(\overline{E_{D}^{B}}\right)$. As previously, this requires measuring the cross section for removing a $\mathrm{B}$ atom in pristine h-BN $\left(\sigma^{B}\right)$, but this time we consider that all the $B$ atoms under irradiation have the same probability to be ejected [15]. The large areas with adsorbates were not considered because the presence of amorphous materials obviously protects the $\mathrm{BN}$ sheet. Therefore, we have

$$
\sigma^{B}=\frac{D_{\mathrm{NP}}}{D_{B} \times d}
$$

with $D_{\mathrm{NP}}$ the density of nanopores, $D_{B}$ the density of B atoms in the $\mathrm{BN}$ sheet, and $d$ the cumulative dose. As $D_{B}$ is a constant (19 at. $/ \mathrm{nm}^{2}$ ) here, we expect $D_{\mathrm{NP}}$ to be proportional to $d$. We measured $D_{\mathrm{NP}}$ as a function of $d$ for three different dose rates. As seen in Fig. S5(e) in Supplemental Material [34], regardless of the dose rate, $D_{\mathrm{NP}}$ increases at the early stage of the growth and systematically decreases when coalescence takes place. Therefore, the measurement of $\sigma^{B}$ requires one to focus on the early stage of the experiments, before coalescence occurs (i.e., below $5 \times 10^{7}$ electron $/ \mathrm{nm}^{-2}$, corresponding to 100 to $160 \mathrm{~s}$ depending on the dose rate). From the linear fitting of the data [Fig. S13(a) in Supplemental Material [34]], we can deduce that the formation rate of monovacancies varies little with the dose rate and ranges between $0.72 \times 10^{-9}$ and $1.11 \times 10^{-9}$ vacancies per electron. Consequently, $\sigma^{B}$ ranges between 0.38 and 0.6 barns and the corresponding $\overline{E_{D}^{B}}$, deduced by using the model of Seitz and Koehler [15,16,35], is between 15.5 and $15.6 \mathrm{eV}$ [Fig. S13(b) in Supplemental Material [34]]. Remarkably, the chemical nature of monovacancies and the nucleation kinetics of nanopores in h-BN under electron irradiation are both confirmed by our DFT calculations that predict an energy of displacement of 15.6 and $17.8 \mathrm{eV}$ for single $\mathrm{B}$ and $\mathrm{N}$ atoms in pristine $\mathrm{h}-\mathrm{BN}$, respectively.

\section{CONCLUSIONS}

In summary, we have combined time-resolved HRTEM and DFT calculations to study quantitatively the nucleation and growth mechanisms of nanopores in h-BN. This coupled approach enabled us to confirm that the growth process is 
carried out in two steps: the creation of a notch on one side of the triangular hole and its expansion via the peeling process. The measured lifetimes of the various edge structures and the structure-dependent $E_{D}^{\text {ed }}$ deduced from DFT calculations show that atom ejection has a lower energy cost during the second step. Moreover, the great majority of atoms are ejected during the peeling process. Also, we also revealed both theoretically and experimentally that atoms are mainly ejected by $\mathrm{B} / \mathrm{N}$ pairs. These features of the growth mechanisms lead to a drastic reduction of mean displacement energy of edge atoms in $\mathrm{h}-\mathrm{BN}$ and allow explaining in a quantitative way the high growth rate of nanopores measured experimentally. The rate and mechanisms of nanopore nucleation that starts with the ejection of single B atoms are also in line with DFT calculations performed on pristine h-BN. This multidisciplinary method can be used in other $2 \mathrm{D}$ materials, but the theoretical determination of $\overline{E_{D}}$ must take into account the whole nucleation and growth processes of nanopores and not only the displacement of edge atoms in a few edge configurations. Interestingly, we have identified irradiation conditions where the size of nanopores increases linearly with the cumulative dose (for perimeters up to $9 \mathrm{~nm}$ ), thus providing a way to produce controlled defects in h-BN. In conclusion, the present paper provides a rational understanding of the key factors that govern the formation of nanopores, paving the way for rational strategies towards engineering $2 \mathrm{D}$ material defects.

\section{ACKNOWLEDGMENTS}

We gratefully acknowledge the financial support of the Region Ile-de-France (convention SESAME E1845 for the JEOL ARM 200F electron microscope installed at the University of Paris). This work was supported by the French National Research Agency through the Project GoBN (Grant No. ANR14-CE08-0018). Y.H.L. acknowledges financial support by the Institute for Basic Science (Grant No. IBS-R011-D1) and K.K.K. acknowledges support from a National Research Foundation of Korea grant funded by the Korea government (MSIT) (Grant No. 2018R1A2B2002302). The research leading to these results has also received funding from the European Union Seventh Framework Program under Grants No. 696656 GrapheneCore1 and No. 785219 GrapheneCore2.
[1] C. Jin, F. Lin, K. Suenaga, and S. Iijima, Phys. Rev. Lett. 102, 195505 (2009).

[2] J. C. Meyer, A. Chuvilin, G. Algara-Siller, J. Biskupek, and U. Kaiser, Nano Lett. 9, 2683 (2009).

[3] N. Alem, R. Erni, C. Kisielowski, M. D. Rossell, W. Gannett, and A. Zettl, Phys. Rev. B 80, 155425 (2009).

[4] R. F. Egerton, Ultramicroscopy 127, 100 (2013).

[5] N. Alem, R. Erni, C. Kisielowski, M. D. Rossell, P. Hartel, B. Jiang, W. Gannett, and A. Zettl, Phys. Status Solidi Rapid. Res. Lett. 5, 295 (2011).

[6] G. H. Ryu, H. J. Park, J. Ryou, J. Park, J. Lee, G. Kim, H. S. Shin, C. W. Bielawski, R. S. Ruoff, S. Hong, and Z. Lee, Nanoscale 7, 10600 (2015).

[7] Ç. Ö. Girit, J. C. Meyer, R. Erni, M. D. Rossell, C. Kisielowski, L. Yang, C.-H. Park, M. F. Crommie, M. L. Cohen, S. G. Louie, and A. Zettl, Science 323, 1705 (2009).

[8] A. V. Krasheninnikov and F. Banhart, Nat. Mat. 6, 723 (2007).

[9] H. Arjmandi-Tash, L. A. Belyaeva, and G. F. Schneider, Chem. Soc. Rev. 45, 476 (2016).

[10] W. Yuan, J. Chen, and G. Shi, Mater. Today 17, 77 (2014).

[11] M. Schleberger and J. Kotakoski, Materials 11, 1885 (2018).

[12] S. M. Gilbert, G. Dunn, A. Azizi, T. Pham, B. Shevitski, E. Dimitrov, S. Liu, S. Aloni, and A. Zettl, Scientific Reports 7, 15096 (2017).

[13] T. Pham, A. L. Gibb, Z. Li, S. M. Gilbert, C. Song, S. G. Louie, and A. Zettl, Nano Lett. 16, 7142 (2016).

[14] O. Cretu, Y.-C. Lin, and K. Suenaga, Micron 72, 21 (2015).

[15] J. C. Meyer, F. Eder, S. Kurasch, V. Skakalova, J. Kotakoski, H. J. Park, S. Roth, A. Chuvilin, S. Eyhusen, G. Benner, A. V. Krasheninnikov, and U. Kaiser, Phys. Rev. Lett. 108, 196102 (2012).

[16] C. J. Russo and J. A. Golovchenko, PNAS 109, 5953 (2012).

[17] A. V. Krasheninnikov and K. Nordlund, J. Appl. Phys. 107, 071301 (2010).
[18] J. Kotakoski, C. H. Jin, O. Lehtinen, K. Suenaga, and A. V. Krasheninnikov, Phys. Rev. B 82, 113404 (2010).

[19] J. S. Kim, K. B. Borisenko, V. Nicolosi, and A. I. Kirkland, ACS Nano 5, 3977 (2011).

[20] A. Govind Rajan, K. S. Silmore, J. Swett, A. W. Robertson, J. H. Warner, D. Blankschtein, and M. S. Strano, Nat. Mat. 18, 129 (2019).

[21] J. Ryou, J. Park, and S. Hong, Nanoscale Res. Lett. 12, 445 (2017).

[22] J.-H. Park, J. C. Park, S. J. Yun, H. Kim, D. H. Luong, S. M. Kim, S. H. Choi, W. Yang, J. Kong, K. K. Kim, and Y. H. Lee, ACS Nano 8, 8520 (2014).

[23] L. Gao, W. Ren, H. Xu, L. Jin, Z. Wang, T. Ma, L.-P. Ma, Z. Zhang, Q. Fu, L.-M. Peng, X. Bao, and H.-M. Cheng, Nat. Commun. 3, 699 (2012).

[24] C. Ricolleau, J. Nelayah, T. Oikawa, Y. Kohno, N. Braidy, G. Wang, F. Hue, L. Florea, V. P. Bohnes, and D. Alloyeau, Microscopy 62, 283 (2013).

[25] D. Alloyeau, T. Oikawa, J. Nelayah, G. Wang, and C. Ricolleau, Appl. Phys. Lett. 101, 121920 (2012).

[26] J. M. Soler, E. Artacho, J. D. Gale, A. García, J. Junquera, P. Ordejón, and D. Sánchez-Portal, J. Phys.: Condens. Matter 14, 2745 (2002).

[27] J. H. Los, J. M. H. Kroes, K. Albe, R. M. Gordillo, M. I. Katsnelson, and A. Fasolino, Phys. Rev. B 96, 184108 (2017).

[28] J. E. Barrios-Vargas, B. Mortazavi, A. W. Cummings, R. Martinez-Gordillo, M. Pruneda, L. Colombo, T. Rabczuk, and S. Roche, Nano Lett. 17, 1660 (2017).

[29] A. V. Krasheninnikov, F. Banhart, J. X. Li, A. S. Foster, and R. M. Nieminen, Phys. Rev. B 72, 125428 (2005).

[30] K. Nordlund, J. Nucl. Mater. 520, 273 (2019).

[31] B. Dacus, B. Beeler, and D. Schwen, J. Nucl. Mater. 520, 152 (2019).

[32] A. Zobelli, A. Gloter, C. P. Ewels, G. Seifert, and C. Colliex, Phys. Rev. B 75, 245402 (2007). 
[33] F. Banhart, Rep. Prog. Phys. 62, 1181 (1999).

[34] See Supplemental Material at http://link.aps.org/supplemental/ 10.1103/PhysRevMaterials.4.014005 for 13 additional figures and a video of h-BN under electron irradiation.

[35] F. Seitz and J. S. Koehler, in Solid State Physics, edited by F. Seitz and D. Turnbull (Acadimic Press, New York, 1956), pp. 307-442.
[36] Q. M. Ramasse, R. Zan, U. Bangert, D. W. Boukhvalov, Y.-W. Son, and K. S. Novoselov, ACS Nano 6, 4063 (2012).

[37] G. T. Leuthner, S. Hummel, C. Mangler, T. J. Pennycook, T. Susi, J. C. Meyer, and J. Kotakoski, Ultramicroscopy 203, 76 (2019).

[38] C. Kisielowski, P. Specht, D. Alloyeau, R. Erni, and Q. Ramasse, AIP Conf. Proc. 1173, 231 (2009). 